\title{
Thoracic Aortic Laceration Resulting in Death, Six Days after a Motor Vehicle Accident: A Case Study
}

\author{
Shravana Kumar Jyoti ${ }^{1^{*}}$, Kazmi $\mathrm{M}^{2}$, Jagadeesh $\mathrm{NR}^{3}$, Byragani $\mathrm{D}^{2}$, Haque $\mathrm{S}^{2}$ and Kotler $\mathrm{WM}^{2}$ \\ ${ }^{1}$ Departments of Laboratory and Forensic Medicine, Health Services Authority Hospital(CIHSA) George Town, Grand Cay- \\ man, Cayman Islands \\ ${ }^{2}$ St. Matthew's University School of Medicine, Grand Cayman, Cayman Islands \\ ${ }^{3}$ Faculty, St. Matthew's University School of Medicine, Grand Cayman, Cayman Islands
}

${ }^{*}$ Corresponding author: Shravana Kumar Jyoti, Departments of Laboratory and Forensic Medicine, Health Services Authority Hospital (CIHSA) George Town, Grand Cayman, Cayman Islands, Fax: 1345- 945-1364, Tel: 1 345-917-3289, E-mail: shravanpreeti@yahoo.co.in

Citation: Shravana Kumar Jyoti, Kazmi M, Jagadeesh NR, Byragani D, Haque S, et al. (2017) Thoracic Aortic Laceration Resulting in Death, Six Days After A Motor Vehicle Accident: A Case Study. J Forensic Crime 1: 106

Article history: Received: 28 September 2017, Accepted: 18 December 2017, Published: 19 December 2017

\begin{abstract}
Blunt traumatic aortic rupture (BTAR) resulting from intimal fracture following a motor vehicle accident is quite rare. Although a standard chest $\mathrm{X}$-ray is an initial gold standard screening test, the diagnosis of aortic rupture requires confirmation by special radiological investigations to identify those at risk for lacerations that are minor or incomplete that can progress to fatal aortic ruptures. Survival depends on early and accurate diagnosis with prompt surgical treatment. Here is an unfortunate death of a 64 year old female's demise due to delayed rupture of aorta; 6 days post Motor Vehicle Accident (MVA) while restrained by a seatbelt in the passenger seat. Reporting this case study will ultimately increase awareness of emergency physicians and trauma teams in patients $>65$ that have been in a MVA causing front impact trauma as a result of chest compression and rapid deceleration forces even when airbags and/or seat belt restraints are in use. In the absence of typical clinical manifestations, prompt identification of an intimal fracture or incomplete tear of the aorta may be identified by subtle symptoms such as upper back pain. If identified early, appropriate surgical intervention can lead to improved patient survival and decreased mortality.
\end{abstract}

Keywords: Blunt traumatic aorta rupture (BTAR); Motor Vehicle Accident and delayed rupture of aorta; Intimal Tear of Aorta; Myxoid Degeneration; Seat Belt trauma

\section{Introduction}

Blunt traumatic aorta rupture (BTAR) is a major cause of death following motor vehicle accidents (MVA). Around 7500-8000 victims die of this condition each year, in the USA and Canada, With the majority of cases being a direct result of an automotive accident, representing approximately $15 \%$ of fatalities involving MVA's in North America [1]. In addition, during a MVA there is rapid deceleration of the thorax and compression of the diaphragm, resulting in extreme torque of the aorta and compression at points of attachment: the sinuses of Valsalva, the isthmus, and the diaphragm [2]. Restraint use, air bag deployment and sudden impact may cause compression of the mediastinum, displacing the heart and giving rise to intraluminal hypertension resulting in rupture through the intima, often continuing into the media and adventitial layers. Complete rupture of the aorta usually results in death at the accident scene, whereas patients with a contained hematoma or incomplete rupture may survive to reach the hospital. Since the later may be true, emergency room physicians and surgical trauma teams must maintain suspicion in order to make a prompt diagnosis. Survival depends on early diagnosis and emergency interventions underscored by studies that indicate $40 \%$ of patients die within 24 hours if surgery is not performed [2].

Death six days following high speed traffic accidents with both direct and deceleration trauma is relatively rare. Using the present case study, the authors would like to raise awareness about performing accurate radiological investigations, in patients with subtle un-retractable back pain, in addition to CT scanning and x-ray of the thorax to diagnose contained hematoma from incomplete ruptures. 


\section{Clinical Case Study Information}

The deceased was a 64-year-old white female. She was the restrained front passenger of a vehicle that crashed head on with a pickup truck. The vehicle was said to be travelling at 20-30miles/hr speed and was a left hand drive. Immediately after the accident, patient was conscious and taken to the local hospital for work-up due to complaints of pain in her head, chest, upper back and abdomen. The examination revealed a conscious and well oriented female with oblique abrasion to upper right chest measuring 7.3 $\mathrm{cm}$. Ecchymotic areas were found across chest and left upper abdomen consistent with seat belt restraint. Vital signs were all stable and her past clinical history was unremarkable. There were no other signs of injuries.

Initial x-ray revealed fracture of right clavicle, lateral1/3.

On day 2: CT scan revealed 5-7 right ribs fracture with small pneumothorax and confirmed clavicle fracture. It was a routine CT scan without the contrast. Since the pneumothorax was small the condition was conservatively managed

Day 3: X-ray showed persisting small pneumothorax on right side

Day 4: patient was clinically well with subsequent x-ray showing marginal improvement of pneumothorax. Plan to continue with conservative treatment was continued. Blood pressure was overall normal throughout her stay in the hospital.

Day 5: patient looked better, began to ambulate, and was breathing easier. Later on she complained of upper back ache which was treated by application of local pain killer ointments.

On the morning of day 6, patient went to radiology to repeat x-ray on a wheel chair. She started gasping, collapsed and became unresponsive. All attempts for resuscitation were unsuccessful and the patient was declared dead a short time later. Clinical diagnosis of pulmonary embolism was made and postmortem was requested.

\section{Forensic Findings}

The postmortem anatomic examination revealed a symmetrical chest with diffuse hemorrhagic areas around right clavicle with a displaced fracture between medial $2 / 3$ and lateral $1 / 3$. No evidence of pneumothorax was seen. The right pleural cavity revealed hemorrhagic fluid with blood clot amounting to $1000 \mathrm{cc}$ and left pleural cavity with $500 \mathrm{cc}$ of the same type of fluid. Hemorrhage was arising from the posterior mediastinum around the aorta. Close and careful dissection at this area showed descending part of the thoracic aorta showing a linear and complete tear measuring $10 \mathrm{~cm}$ in the posterior aspect with the irregular margins of the vessel wall. The tear involved all the layers and it's supporting fibrous tissue which adhered aorta to the thoracic vertebrae (Figure 1). There was no aneurysmal dilatation or transverse intimal slit which is usually seen in immediate deaths due to aortic lacerations. Histology of the aorta at this area showed myxoid degeneration of the media. Heart weighed 240 grams with patent coronaries. No signs of atherosclerosis were noted. The stomach, small and large intestines, pancreas and liver were all in their usual anatomical conditions.

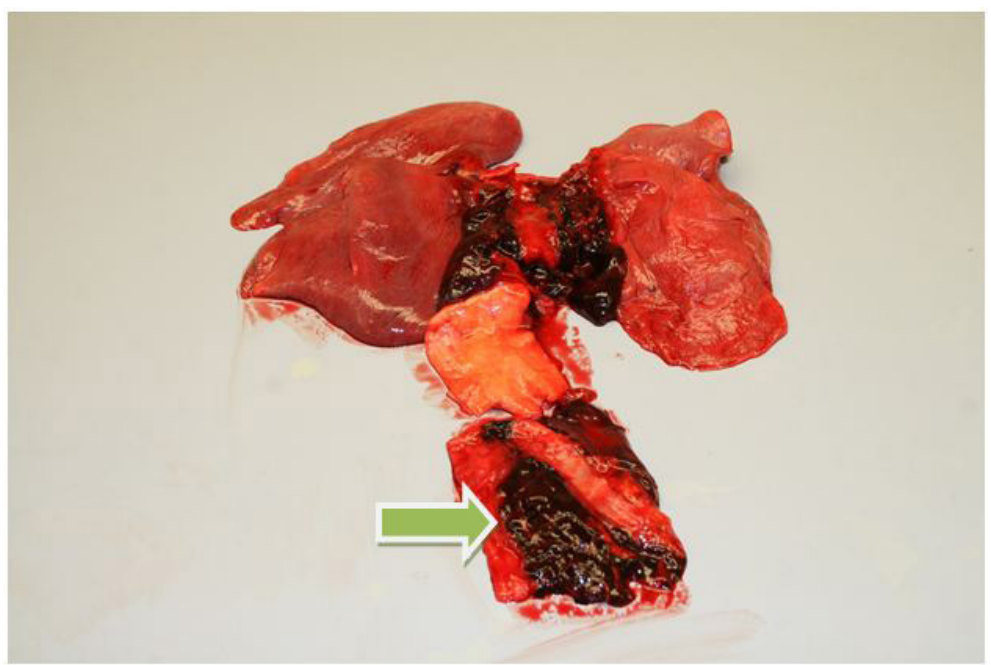

Figure 1: Thoracic aorta showing complete tear on the posterior aspect

\section{Clinico-pathologic Correlation}

The pathologist was confronted with this unique case. The autopsy findings were correlated with the medical history and clinical data. It revealed that she died from rupture of the aorta due to the laceration sustained during the motor vehicle accident which was 6 days prior to her death. The laceration was on the posterior aspect of the aorta which itself was supported by the ligamentum arteriosum to the dorsal spine. The fatal injury to the vessel appeared to be due to the front impact trauma as a result of chest compression and rapid deceleration, transmitting the impact trauma force through the chest wall, through the heart, to the aorta. 
This injury is consistent with significant chest compression seen in MVA's in restrained passengers. It is likely that the front impact injury to the vessel wall was sustained during systole of the heart. The initial lesion may have been a minor intimal tear, well supported by soft tissue at the back of the aorta and progressed to a full tear over the subsequent days or when she became ambulatory on day $5^{\text {th }}$ of admission to the hospital. The fatal injury was deemed a delayed consequence of the motor vehicle accident.

\section{Discussion}

Radiographic chest films and CT scans with and without contrast have been extensively used as a method of screening for aortic rupture. Markers of mediastinal hematoma associated with aortic rupture on the chest x-ray may not initially be present. These include: a widened mediastinum, blurred aortic knob, loss of concavity of the aortopulmonary window, left apical extra pleural cap, displacement of the nasogastric tube, tracheal deviation, and left hemothorax [3]. Fractures of the first and second ribs, clavicle, and sternum identified on $\mathrm{x}$-ray are also resultant of front impact trauma and are associated with aortic injury. Taken individually or in combination, these findings lack sufficient sensitivity and specificity to consistently identify patients with aortic rupture. At best, the chest $\mathrm{x}$-ray film is a useful initial screening test to determine which patients with trauma require more definitive testing and confirmation by aortography, computed tomography, or magnetic resonance imaging before subsequent surgical intervention $[4]$.

Based on the mechanism and severity of injury and the findings on the chest radiograph, many trauma centers proceed to the diagnostic standard for aortic rupture, aortography. Gundry, et al. have recommended that all patients over 65 years of age who have thoracic trauma undergo aortography, because of the higher prevalence of aortic transaction in this age group [5]. Data indicate that the use of transesophageal echocardiography can also be used in the emergency evaluation of patients with blunt chest trauma, when the risks of aortography are great [5-7]. In a recent article, computed tomography was used to classify aortic injuries on the basis of severity: grade I, intimal tear; grade II, intramural hematoma; grade III, aortic pseudo aneurysm; and grade IV, free rupture. From these classified aortic lesions, patients with minimal aortic injuries (grades I and II) may be managed medically, with the majority resolving within 8 weeks [8].

The results of a careful review of the literature do not support further studies in patients with fracture of the ribs after motor vehicle accident, and no other clinical manifestations of blunt force trauma. In patients suffering blunt trauma, the risk of disruption of the aorta is not greater in patients with fracture of the upper two ribs, compared with victims of trauma with fracture of other ribs or those without fracture of ribs [6]. However, clinical manifestations are often absent initially in patients with disruption of the aorta, but evidence of mediastinal hemorrhage is almost always present on roentgenograms of the chest [5].

Careful consideration of the symptoms should be done in patients who survive. The symptoms that appear minor but could turn out to be fatal include unusual pan, late onset pain, persistent and pain associated with rib fractures. Any back pain which appears late should be explored to rule out progressive tear. Simple examinations like arterial blood pressure and ECG examinations can be supportive to rule out cardiac causes.

The co-operative crash injury study by D. Richens, et al. revealed 74 out of 132 occupants of vehicles involved in pure frontal impact, with BTAR cases were known to be wearing a seat belt restraint methods [6]. These findings show that the use of a belt or does not eliminate the risk of BTAR but rather increases it. The location of aortic rupture in unbelted victims was more often in the ascending aorta, especially in drivers, whereas in seat belt wearers the distal descending aorta was statistically more often ruptured, especially in front passengers. Some interior part of the car was the most common cause of fatal thoracic impacts in seat belt wearers. The main mechanism leading to rupture of the ascending aorta seems to be a severe blow to the bony thorax. Ruptures of the distal descending part of the aorta were frequently associated with fractures of the thoracic vertebra [7].

\section{Conclusion}

The value of this patient's thorough and skillfully performed autopsy is greatly diminished if the findings and correlations are not adequately communicated to end users of this article. Blunt traumatic aortic rupture resulting from intimal fracture following a motor vehicle accident is quite rare but still the possibility exists as is seen in this case. Survival depends on early and accurate diagnosis with prompt surgical treatment. Although standard chest radiography is useful as an initial screening test, the diagnosis of aortic rupture requires confirmation by aortography, computed tomography, or magnetic resonance imaging may be required to identify those at risk for lacerations that are minor or incomplete that can progress to fatal aortic ruptures. It behooves emergency physicians and trauma teams to take into account patients age $>60-65$, along with subtle symptoms i.e. upper back ache, to determine if further testing is necessary in the absence of typical clinical manifestations of BTAR, in patients with MVA with rapid deceleration forces when airbags or seat belt restraints were in use. Prompt identification of an intimal fracture or incomplete tear followed by appropriate surgical intervention can lead to improved patient survival and decreased mortality.

\section{References}

1. Fabian TC, Richardson JD, Croce MA, Smith JS, Rodman G, et al. (1997) Prospective study of blunt aortic injury: multicenter trial of the American Association for the Surgery of Trauma. J Trauma 42: 374-83.

2. Woodring JH, Dillon ML (1984) Radiographic manifestations of mediastinal hemorrhage from blunt chest trauma. Ann Thorac Surg 37: 171-8. 
3.Metaxa V, Tsagourias M, Matamis D (2011) The role of echocardiography in the early diagnosis of the complications of endovascular repair of blunt aortic injury. J Crit Care 26 : 434.e7-12

4. Sanchez-Ross M, Anis A, Walia J, Randhawa P, Esrig BC, et al. (2006) Aortic rupture: comparison of three imaging modalities. Emerg Radiol 13: 31-3.

5. Gundry SR, Williams S, Burney RE, MacKenzie JR, Cho KJ (1983) Indications for aortography: radiography after blunt chest trauma: a reassessment of the radiographic findings associated with traumatic rupture of the aorta. Invest Radiol 18: 230-7.

6. Richens D, Kotidis K, Neale M, Oakley C, Fails A (2003) Rupture of the aorta following road traffic accidents in the United Kingdom 1992-1999. The results of the co-operative crash injury study. Eur J Cardiothorac Surg 23: 143-8.

7. Ripple MG, Grant JR, Mealey J, and Fowler DR. Evaluation of Aortic Injury in Driver Fatalities occurring in Motor Vehicle Accidents in the State of Maryland from 2003-2004.

8. Sandhu HK, Leonard SD, Perlick A, Saqib NU, Miller CC (2017) Determinants and outcomes of nonoperative management for blunt traumatic aortic injuries. J Vasc Surg 5214: 32024-4. 